\title{
ON PROBLEMS WITH SOLUTION-DEPENDENT LOAD
}

\author{
ROMAN BOGACZ \\ Warsaw University of Technology, Faculty of Automative and Construction Machinery Engineering, Warsaw, Poland
}

\section{Kurt Frischmuth}

University of Rostock, Institute of Mathematics, Rostock, Germany

e-mail: kurt.frischmuth@uni-rostock.de

\begin{abstract}
In Continuum Physics, general balance problems between internal fluxes and external sources are considered, mostly on some domain $\Omega$ called a material body. Typically, the fluxes are determined by a wanted field - the solution or configuration of the body, possibly augmented by derivatives or other suitable quantities specifying the state of the system. In order to obtain a closed problem formulation, initial and boundary conditions need to be provided as well. In this paper, we discuss some issues appearing in the case that the sources, e.g. forces in a mechanical problem, are not known in advance, but related to the wanted configuration in terms of values and/or derivatives of the solution. An analogy between thermodynamic and mechanical problems is shown.
\end{abstract}

Keywords: feedback, dynamics, stabilty, columns, follower forces

\section{Introduction}

Let us consider a problem of the general form

$$
H_{t}=-\operatorname{div} F+G
$$

Here, by $F$ the flux of some balance quantity is denoted, $G$ is a corresponding source term, and $H$ is the storage of the quantity, which may be scalar or vector-valued. All dependent fields are functions of a space variable $x$ and time $t$.

As an example, consider internal energy $\epsilon=c \vartheta$ with temperature $\vartheta$, heat flux $q$ and heat sources $r$, cf. (Kosiński and Frischmuth, 2001), which are related to each other by

$$
\epsilon_{t}=-\operatorname{div} q+r
$$

In order to find a solution, a constitutive relation for the flux $q$ is introduced by Fourier's law

$$
q=-\kappa \nabla \vartheta
$$

This leads to the partial differential equation

$$
c \vartheta_{t}=\operatorname{div}(\kappa \nabla \vartheta)+r
$$

with the heat capacity $c>0$ and the heat conductivity $\kappa>0$.

For this equation, one considers initial-boundary value problems with given sources $r=r(x, t), x \in \Omega, t \geqslant 0$, given boundary values $\vartheta(x, t)=\bar{\vartheta}(x, t), x \in \partial \Omega, t \geqslant 0$, and a given initial temperature field $\vartheta(x, 0)=\vartheta_{0}(x), x \in \Omega$. 
The above problem is well posed, i.e., it has a unique solution, which depends continuously on the data. Instead of prescribed values of the unknown function $\vartheta$ at the boundary, the heat flux $q_{n}=-\kappa \nabla \vartheta \cdot \vec{n}=-\kappa d \vartheta / d \vec{n}$ may be given. The first case is called Dirichlet conditions, in the second one we speak about a Neumann boundary. There may be a part of the boundary, where Dirichlet conditions are prescribed, while on the remaining part Neumann conditions apply.

In this paper, our focus is on a third type of boundary conditions, the Robin condition, which generalizes both previous cases

$$
q_{n}=-\kappa \frac{d}{d \vec{n}} \vartheta=\gamma\left(\vartheta-\vartheta_{\text {env }}\right)
$$

Here $\vartheta_{\text {env }}$ is a given temperature of the environment, and $\gamma \geqslant 0$ is the conductivity of a (virtual) layer between the considered body, occupying the domain $\Omega$, and the surroundings. This means that the boundary values of $\vartheta$ are no longer known in advance but depend on the normal derivative of the solution $\vartheta$.

Notice that also the assumption of known in advance heat sources $r$ may be not adequate. The intensity of local heating may be a function of temperature, for instance as a result of thermo-chemical coupling, if the kinetics of an exothermal reaction depends on temperature, i.e., $r=r(x, t, \vartheta(x, t))$. In lower-dimensional problems, arising from models of a plate, shell or beam, again the exchange with the environment might be the dominant issue. In such cases, a higher value of the solution leads to a lower source, e.g. by a law of the form $r=\delta\left(\vartheta_{\text {env }}-\vartheta\right)$. In the reaction case, the opposite effect may lead to an unstable situation: the higher the temperature, the faster it grows. It is a challenge not only to solve an initial-boundary value problem for heat transfer problem (1.4) with the given specifications, but also to find stationary solutions and to determine whether they are stable.

When it comes to mechanics of continua, in the general 3D case, the unknown real valued function $\vartheta$ is replaced by a vector field $\mathbf{u}=\mathbf{u}(x, t) \in R^{3}$, the role of the heat flux is taken by the tensor $\mathbf{T}$, which comprises the stresses, and the balanced quantity is the linear momentum $\rho \mathbf{u}_{t t}$. The constitutive law, in the simplest elastic case, is given by a linear dependence between strain and stress, with the strain calculated from the gradient of $\mathbf{u}$, cf. (Bogacz and Janiszewski, 1985). Equation (1.1) takes here the form

$$
\rho \mathbf{u}_{t t}=-\operatorname{div} \mathbf{T}+\mathbf{b}
$$

with the body forces $\mathbf{b}$ playing the role of the former $r$. A typical, linear, homogeneous and isotropic relation for $\mathbf{T}$ is

$$
\mathbf{T}=2 \mu \varepsilon+\lambda \operatorname{tr}(\varepsilon) \mathbf{I}
$$

where $\mathbf{I}$ is the identity (unit tensor), $\mu$ and $\lambda$ are positive constants (Lamé moduli), and $\varepsilon$ is the tensor of (small) deformations

$$
\varepsilon=\frac{1}{2}\left[\nabla \mathbf{u}+(\nabla \mathbf{u})^{\mathrm{T}}\right]
$$

More general situations, anisotropy, large deformations, inelastic materials, etc. are important issues, but out of the focus of this article - we restrict ourselves to linear models, valid in the neighborhood of equilibrium states.

There is one feature common for the thermodynamic and the mechanical master model. Again, the body forces as well as the boundary conditions, i.e. the interface between our model and the environment, may depend on the solution, cf. (Beck, 1952; Timoshenko, 1921). Furthermore, the dependence may be stabilizing, destabilizing, or switch from stable to unstable at certain states. And once more, it will be a challenge to find dynamical solutions, distinguish stationary 
ones, and resolve the stability issue, cf. (Bogacz and Frischmuth, 2012; Bogacz et al., 1980, 2008; Bogacz and Janiszewski, 1985; Preumont and Seto, 2008; Ringertz, 1994).

On the other hand, the big difference is that now, in mechanics, second derivatives with respect to time enter the evolution equation.

Since we concentrate on solutions close to a natural state of rest, the assumptions of small deformations (geometric linearity) and material linearity are reasonable. Additionally, we also postulate linearity of the feedback relation between body forces and boundary conditions and the wanted vector field $\mathbf{u}=\mathbf{u}(x, t)$. Due to linearity, the setup

$$
\mathbf{u}(x, t)=\mathbf{w}(x) \mathrm{e}^{\mathrm{i} \omega t}
$$

is known to work in the context of mechanics. The first order derivatives in the evolution equation are just scalar multiples of the unknown function, and the second order derivatives satisfy

$$
\mathbf{u}_{t t}(x, t)=-\omega^{2} \mathbf{u}(x, t)
$$

Using this, the task reduces to an operator eigenproblem of the form

$$
\mathbf{L w}=-\omega^{2} \rho \mathbf{u}(x, t)
$$

where the eigenvalue is $-\omega^{2}$. The linear operator is composed of two contributions, one corresponds to the stiffness of the classical problem, the other one represents the feedback connected with the solution dependence of the loads. This will be discussed in more detail in the next Section.

After a suitable discretization, e.g. by the FEM or by FDM, a matrix eigenvalue problem emerges, modes of oscillation may be found and superposed. This way, initial conditions may be met and hence initial-boundary value problems solved. A discussion of the real and imaginary parts of the spectrum determines stability of the trivial solution $\mathbf{w}=\mathbf{0}$.

It should be mentioned that other setups are successful as well, e.g. $\exp (\omega t)$ or $\sin (\omega t)$ lead to the same results, bearing in mind Euler's formulas. In the thermodynamic case, where there is only a first order time derivative, obviously $\exp (\omega t)$ is more convenient, while in the mechanical case, when it comes to damping, the exponential representations are advantageous.

\section{A classic case}

A particular example of the considered class of problems is the 1D Bernoulli-Euler beam model with special loading conditions. Due to the low dimensionality of the domain and due to the fact that the complete solution is represented by a single scalar function, i.e. by the displacement of the middle surface of the beam, this problem is well suited for a qualitative study. For a discussion of the assumptions and applicability of the Bernoulli-Euler beam theory we refer to (Timoshenko, 1921; Tomski et al., 1996).

The equation of balance of linear momentum, after substituting the assumed constraints, takes the form

$$
\left(S(x) u_{x x}(x, t)+P u(x, t)\right)_{x x}+k(x) u(x, t)=-\rho(x) u_{t t}(x, t)
$$

Here, $S$ is the bending stiffness (related to the elastic moduli and the cross section of the beam, see Bogacz and Janiszewski (1985)), $P$ a constant compressing axial force, $x \in[0, l]$ the scalar space variable (position), $\rho$ the mass density (per unit length, i.e. the $3 \mathrm{D}$ material density multiplied by the area of the cross section). The role of $k$ is the solution dependence of the load it is well-known as the Winkler constant. Similarly, as in the heat source case, we have a lateral force $-k(x) u(x, t)$, which pushes the displaced beam back to its trivial position. 
As a generalization of (2.1), an analogous contribution due to viscous damping may be added (Frischmuth et al., 1993), so that the lateral force from the support becomes $-k(x) u(x, t)-$ $v(x) u_{t}(x, t)$.

Problems of this class have a great practical relevance in several fields of engineering, see e.g. (Kaliski and Solarz, 1962; Kerr, 1988; Przybyłowicz, 2008; Bogacz et al., 2014).

Introducing, as before, a product setup, this way splitting the time and space dependencies in the form of a standing wave, the heart of the matter will be again an eigenproblem of the form

$$
\mathbf{L}_{1} \mathbf{w}+P \mathbf{L}_{2} \mathbf{w}+k \mathbf{w}=\rho \omega^{2} \mathbf{w}
$$

The name of the eigenvalue, i.e. $\omega^{2}$, is no obstacle, as long as $\omega$ alone does not enter the equation. This happens, however, if damping is introduced, so that (2.2) becomes

$$
\mathbf{L}_{1} \mathbf{w}+P \mathbf{L}_{2} \mathbf{w}+k \mathbf{w}+\mathrm{i} \omega v \mathbf{w}=\rho \omega^{2} \mathbf{w}
$$

This quadratic eigenproblem may be transformed into a common one for the pair $(\mathbf{w}, \mathbf{v})$, where $\mathbf{v}$ stands for the velocity, i.e. $\mathbf{v}=\mathrm{i} \omega \mathbf{w}$. With this definition, (2.3) becomes

$$
\begin{aligned}
\mathbf{v} & =\mathrm{i} \omega \mathbf{w} \\
-\frac{1}{\rho}\left(\mathbf{L}_{1} \mathbf{w}+P \mathbf{L}_{2} \mathbf{w}+k \mathbf{w}+v \mathbf{v}\right) & =\mathrm{i} \omega \mathbf{v}
\end{aligned}
$$

Now, after discretization, $\mathbf{L}_{1}$ and $\mathbf{L}_{2}$ become matrices, and $(\mathbf{w}, \mathbf{v})$ will be represented by $n$ nodal values of the displacement and $n$ more scalar values for the lateral velocities.

The solution of the problem depends in a very sensitive way on the boundary values imposed on the displacement. For a fourth order boundary value problem on a finite interval $[0, l]$, the most straightforward choice is to prescribe zero displacements and zero (space-) derivatives at both ends. This describes a clamped-clamped situation. In the spirit of solution dependence, however, other choices are of greater interest. While at $x=0$ we demand clamped conditions, at the other end, $x=l$, various linear relations between the angle of inclination $u_{x}(l, t)$, bending moment $S(l) u_{x x}(l, t)$, lateral force $-\left(S(x) u_{x x}(x, t)+P u(x, t)\right)_{x}$ at $x=l$ may be considered.

Two special cases gained in the past a great deal of attention. One of them is based on the assumption that a lateral force proportional to the axial load $P$ and to the angle of inclination $u_{x}(l, t)$ is applied at $x=l$. Indeed, this fits into the class of problems with a load dependent on the solution (and its derivatives). If the coefficient is negative, the force is, in fact, stabilizing. If chosen equal to -1 , the boundary condition simplifies to $\left(S(x) u_{x x}(x, t)\right)_{x}=0$ at $x=l$. In the case of constant $S$, just the third order derivative is set equal to zero. This condition is usually combined with that of a vanishing bending moment, which means a zero second order derivative. Together, these boundary conditions and the equations of motion define Beck's model of a compressed column (Beck, 1952). The second of the mentioned special cases is Reut's column, which is characterized by a vanishing lateral force and a solution dependent bending moment at $x=l$ proportioanal to $P$ and to the displacement of the tip, $S(l) u_{x x}(l, t)=-P u(l, t)$, cf. (Imiełowski and Mahrenholtz, 1997).

\section{Mathematical techniques}

In order to solve problems of the discussed class, some analytical background is needed (Euler, 1778a,b); however, eventually, only computational methods give quantitative answers, cf. for instance (Bogacz and Frischmuth, 2012; Bogacz et al., 2008). Analytical properties of the involved operators play a role for qualitative studies, e.g. whether they are coercive or self-adjoined. This decides about the localization of the requested spectrum in (2.4). 
The two most essential numerical considerations concern the questions:

- Up to which value of the loading parameter, in the case of the compressed column this is the value of the force $P$, all solutions are bounded, for instance periodic. In fact, we want to establish stability of the undeformed system at rest.

- Furthermore, when a critical state is reached, what is the form of the displacement that grows out of any limited neighborhood of the trivial state, and what is the way it departs from the state of rest. The later question relates to the effect of flutter, respectively divergent loss of stability.

While in the general case of two- or three-dimensional domains $\Omega$, the method of choice is the discretization of the spatial operator, which leads to a high-dimensional sparse matrix eigenproblem (Frischmuth et al., 1993; Hanaoka and Washizu, 1980; Ringertz, 1994; Tada et al. $, 1985,1989)$, in the one-dimensional case shooting methods are a quite attractive alternative (Bogacz and Frischmuth, 2012; Bogacz and Janiszewski, 1985; Kaliski and Solarz, 1962). Indeed, using explicit formulas for the solution of (2.1) with setup (1.11), the analysis can be reduced to a small matrix problem. This allows an effective and precise calculation of the first eigenmodes and hence the answer to the above formulated questions. This method has been successfully applied e.g. in (Bogacz and Janiszewski, 1985; Kaliski and Solarz, 1962). A weak point of the shooting method is that analytical solutions to the ODE resulting from (1.8), (1.11) and (2.1) are available only in the case of constant coefficients, i.e. prismatic homogeneous beams. To overcome this, segmented columns are studied, which are composed of two or more prismatic pieces. A smooth mass and stiffness distribution would require computational methods. This was done e.g. in (Bogacz et al., 2008) - but the cost increased dramatically. Hence, in the case of a continuously varying cross-section, even a conical one, the shooting method ceases to be efficient.

In this paper, we compare the shooting method - where applicable - with the general approach, based on eigenvalue methods for medium size matrices. This technique is accurate also for higher modes, and it is easily adapted to beams and columns with variable cross-sections. Modifications of the model, e.g. the introduction of supports, elastic or viscous, concentrated or distributed, are extremely easy to implement. Further, elements of the implementation may be re-used for the solution of transient and even nonlinear problems.

The idea of the algorithm is straightforward. We introduce $n$ equally spaced grid points $x_{j}$ along the middle line of the beam/column. Their distance is $h=l / n$. Between the grid points, exactly at $(j-1 / 2) h, j=1,2, \ldots, n-1, n$, we slice the column into cells. To each cell, its mass is assigned on the basis of the given mass density. Further, for given lateral displacements $W_{j}$ at the grid points, by central differences the curvature, and hence the bending moment at $x_{j}$ is calculated as

$$
M_{j}=S_{j} \frac{W_{j-1}-2 W_{j}+W_{j+1}}{h^{2}}
$$

Taking the first order difference of the $M_{j}$-values, adding $P$ times the first order difference of the displacements themselves, one obtains minus the lateral force at the cell boundaries. According to (2.1), allowing for additional lateral support forces if such are present, it is possible to calculate the lateral acceleration with second order accuracy with respect to the step size $h$.

Special attention is to be paid to the first and last nodes. For the first one, $j=1$, we are missing the values $W_{0}$ and $W_{-1}$, which according to (3.1) are needed to obtain $M_{0}$ and $M_{1}$. Here we may use $W_{0}=0$ and $W_{-1}=W_{1}$, which follow from the clamped boundary conditions.

The derivation of the equations of motion for the last two nodes was discussed in detail in (Bogacz et al., 2008). Again, application of difference operators requires nodal values beyond the last grid value. These have to be substituted in accordance with the prescribed boundary conditions. For instance in the Beck case, the moment $M_{n}$ is directly given, the same for Euler 
and Reut. In the latter, the moment depends on $W_{n}$, which still is easy to implement. Conditions involving the lateral force, i.e. the third and the first derivative, cause more trouble. In particular, in the mentioned examples of Beck's and Reut's columns, the matrix representation of the operator $\mathbf{L}=\mathbf{L}_{1}+\mathbf{L}_{2}$ will be nonsymmetric.

In Beck's case, with constant mass density and stiffness, the shooting method leads to the analysis of the root curves of

$$
f(\omega, P)=\operatorname{det}\left[\left[\begin{array}{llll}
0 & 0 & 1 & 0 \\
0 & P & 0 & 1
\end{array}\right] \exp (l \mathbf{A}(\omega, P))\left[\begin{array}{ll}
0 & 0 \\
0 & 0 \\
1 & 0 \\
0 & 1
\end{array}\right]\right]
$$

with

$$
\mathbf{A}(\omega, P)=\left[\begin{array}{cccc}
0 & 1 & 0 & 0 \\
0 & 0 & 1 / S & 0 \\
0 & 0 & -P & -1 \\
-\rho \omega^{2} & 0 & 0 & 0
\end{array}\right]
$$

The zeros of $f$ correspond to pairs of the frequency $\omega$ and the compressing force $P$ for which there are nontrivial solutions to a homogeneous system of equations in terms of the moment and lateral force at the foot of the column, which are mapped to the moment and force at the top.

The matrix exponential function used in (3.2) abbreviates the solution formulas for the system

$$
\mathbf{y}^{\prime}(x)=\mathbf{A}(\omega, P) \mathbf{y}(x)
$$

where $\mathbf{y}=[w, \alpha, M, Q]^{\mathrm{T}}$, meaning the lateral displacement, angle of inclination, bending moment and lateral force. For a given rectangle of interest, the solutions to $f(\omega, P)=0$ with $f$ from (3.2) have the well-known form shown in Fig. 1.

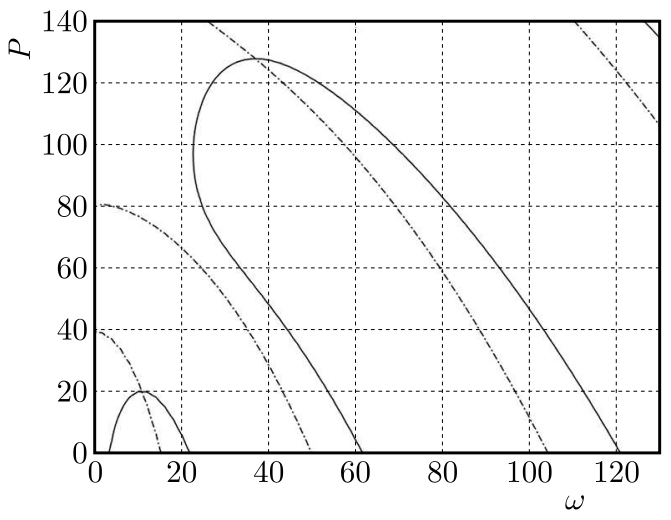

Fig. 1. Root curves in Beck's case, shooting method (density, stiffness and length all equal to 1, dimensionless)

We obtain a critical force of 20.05 together with a critical frequency of 10.87 . At this point, the first frequency arc of the first branch of the zero level-set meets with the second one, which we consider the standard topology. Usually, the third meets the fourth and so on. However, in particular for optimized geometries, this alternating sequence of growing and falling arcs becomes disturbed. An example will be shown in Fig. 6, the right part. Moreover, especially in the case of damping, the frequencies are complex-valued from the start on, so that there is no longer a canonical order by their frequencies. 


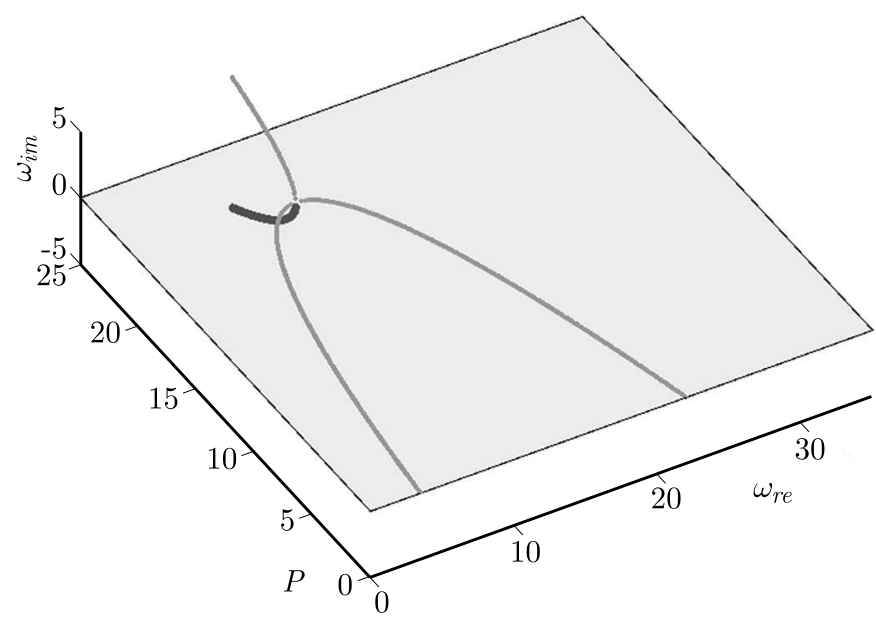

Fig. 2. Eigenfrequencies in dependence on the compressing force. Negative imaginary parts, thick black arc below the 0-plane, indicate instability

The discretization method, followed by an eigenvalue calculation, confirms these values. We used $n=65$ for Fig. 2.

The minimum of the imaginary parts of the spectrum of the matrix representation of (2.2), here with $k=0$, is required to be nonnegative. This is true below the critical value of $P_{c r} \approx 20.03$, and it is violated when $P$ exceeds $P_{c r}$.

\section{Improvements}

Once we are able to find critical states, an obvious goal is the improvement of the situation in the sense that a higher load can be carried by the structure. There are several options to achieve this. A first way to push the limit of stability higher up is to add supports, i.e. to introduce new loads depending on the values of the displacement, which push or pull the column under consideration back to its original position. Technically, such external forces may be exerted by trusses, which can be modelled as linear springs attached to the column. Further, viscous damping elements may be used to stabilize. Both may be applied alone or together, in series or in parallel, concentrated in a single point, in several points, or continuously distributed. For a detailed analysis, we refer to (Bogacz and Janiszewski, 2008) and our forthcoming book.

An alternative way is to change the considered object - in this case the column - itself. In particular, keeping its length constant, the cross section may be varied, and the material may be changed, so that the mass density and the bending stiffness become design variables. Internal damping may be added, e.g. in the form of a layer of a viscous material, so that a bending moment proportional to the rate of change of the curvature enters the equation of motion. Also graded materials have been studied in the context of damping oscillations in columns (Przybyłowicz, 2008).

Finally, in the age of mechatronics, active control of oscillations of a column may be considered. Assuming that the present deformation of the middle line is known, e.g. measured by optical methods, an optimal position for the application of a lateral force may be determined and applied by an actuator.

In the remaining part of this Section, several versions of modified Beck's columns will be analyzed. Our special interest will be in unexpected effects. Indeed, making a column twice as thick will make it much more stable, which is not a surprise, and which is expensive in terms of material cost, volume and weight. We are looking for intelligent and cost-effective alternatives. 


\subsection{Mass reduction at the top end}

We study the sensitivity of the critical load of Beck's column with respect to changes of its shape. In particular, we will show that it is possible to obtain a higher critical load without using more material. It is enough to reduce the cross section in certain segments, or to redistribute mass, taking material away in some parts, attaching it to others. Here two cases are distinguished: scaled similar profiles and profiles of constant depth. In the first case, stiffness is proportional to the fourth power of the scaling parameter, density changes with its square. In the second case, the exponents are three and one. The effects are analogous, so we pick just one as example.

In Fig. 3, the black curves show the root curves for a segmented column, with the upper part of $15 \%$ of the length, reduced in width by $25 \%$. This saves almost $4 \%$ of the material, but gives an increase of the critical load from 20.05 to 21.05. Notice the right-shift of the black lines, with reference to the gray ones, corresponding to the column with constant parameters. This means that not only the critical load, but also the critical (resonance) frequency is increased by the modification.

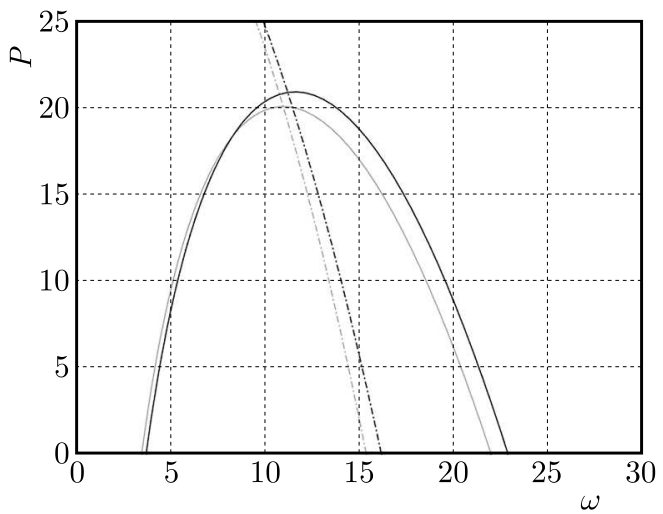

Fig. 3. Improved critical load by reduction of the top segment

It turns out that not much more can be gained by the concept of a two-segment column. A further increase can be obtained, however, by attaching the saved material to the lower segment. Hence, at the same cost of the material, a column with around $8 \%$ higher critical load can be found.

The previous case requires just a study of two parameters: fraction of the lower segment and reduction of width of the upper part. This can be done with reasonable accuracy and effort by calculating a full table. Introducing more segments and widths makes a systematic approach too expensive. Further, the results for columns with three or four segments of variable length are not so promising.

Much better results are obtained by a higher number $N$ of segments of equal length, and optimization of the vector of thicknesses of the corresponding cross sections. This fails at $N=2$, but already at $N=16$ a considerable improvement of the critical load is possible. This level is kept if the segmented column is replaced by a column with continuous width function, interpolating widths of the segment of the optimally segmented column. A detailed analysis can be found in (Bogacz and Frischmuth, 2012) and the forthcoming book by the authors.

Some remarks on the mechanism of loss of stability may be useful for the understanding of the next Subsections. First, reduction of the cross-section of some segment gives a smaller mass density (per unit of length), and also a smaller stiffness against bending. Looking at the form of oscillations - better even watching an animation - one observes a certain lash-back effect. Both changes, that of mass as well as that of stiffness, contribute positively to the increase of the critical load. In fact, using a lighter material of identical stiffness for the upper segment would yield a small improvement of $P_{c r}$, same is true for a material (or profile) of the same density 
and smaller stiffness. The partial improvements would be around $4 \%$ for the change of mass, when keeping the stiffness, and $0.7 \%$ for changing the stiffness while keeping the mass in the upper segment. Notice that the combined effect is considerably larger than their sum. Changing simultaneously the mass density with the width, the stiffness with its third power, we gain more than $5 \%$. Hence, there is something like a cross-activity or interaction between both effects.

If we increase the width of the bottom segment by around four per cent, the total mass will be the same as that of the uniform column. Then, instead of $5 \%$, we already obtain a $20 \%$ improvement of the critical load.

In the case of the upper segment of a geometrically similar shape as that of the lower segment, e.g. in the case of a circular tower, the improvements are about twice as large. For instance, in the case of the unchanged bottom segment, we can obtain more than $8 \%$ saving of the material and more than $9 \%$ higher critical load.

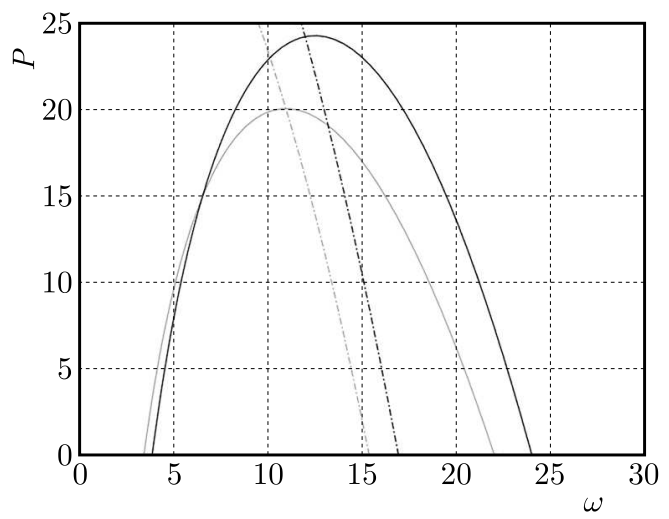

Fig. 4. Increase of the critical load by re-distribution of mass (two segments)

Applying optimization techniques, e.g. an evolution type algorithm, followed by the Nelder-Mead simplex method, columns with $4,6,16, \ldots$ segments can be tuned to support loads of more than five times higher than those of the uniform width column. For the results, e.g. characteristic curves, shapes and forms of oscillation, we refer to our forthcoming book. However, it should be mentioned that the extremely optimized columns are very sensitive to the slightest changes of their geometry (mass and stiffness distributions), to external forces (e.g. even minimal damping), and boundary conditions, see (Ringertz, 1994).

\subsection{Lateral supports}

In this Subsection, we consider the influence of lateral supports in the form of elastic and viscous elements. These may act either concentrated at chosen positions or distributed over the length of the column or, finally, combined in the form of a continuous distribution with Dirac-type atoms.

Of particular interest are viscous dampers. Surprisingly, as opposed to elastic ones, their application turns out to be not as promising as might be expected. As previously, effects of cross-influences may be observed. First, we study the influence of forces proportional to the lateral velocity, with a uniform distribution along the length of the column. We found that the influence of such linear viscous damping forces lifts the eigenvalues in Fig. 2 in the direction of the imaginary axis. The effect on the onset of growing modes of oscillation is very small, because the imaginary parts bifurcate out of the horizontal plane containing the lifted characteristic in a parabolic way, so that the increment of $P_{c r}$ is only second order in the damping coefficient $\nu$. Now, if we try a viscous damper giving a concentrated force in a single position, the result is surprisingly bad: depending on the point, where the damper is attached, its effect will be nil or negative. A damper mounted at an inappropriate distance from the fixed end may destabilize 
Beck's column. However, if we combine uniform and concentrated damping, a combined advantageous effect can be obtained. So for instance, $\nu=1$ results in a critical load higher by $0.29 \%$ than the undamped column, while $\nu=10$ already gives $21 \%$. This is more than for the segmented column of the same mass - but quite difficult to implement in practice. A concentrated damper just below the midpoint of the column at $x=0.48$ gives nothing, but when combined with the small uniform damping $\nu=1$, an increase of $P_{c r}$ by $50 \%$ is possible, see Fig. 5 .

(a)

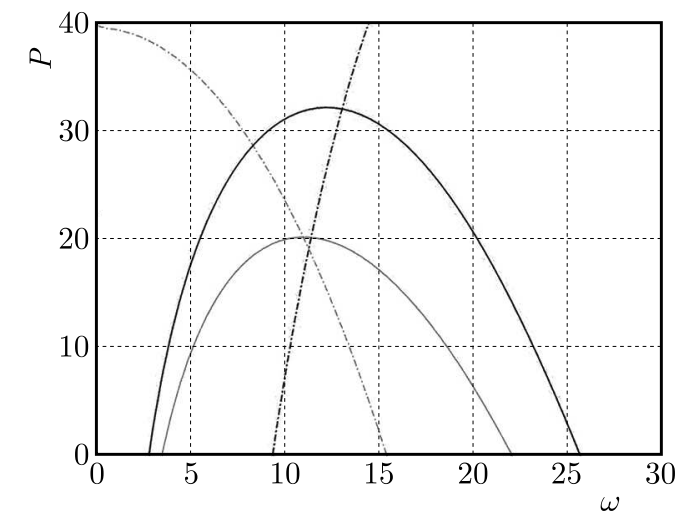

(b)

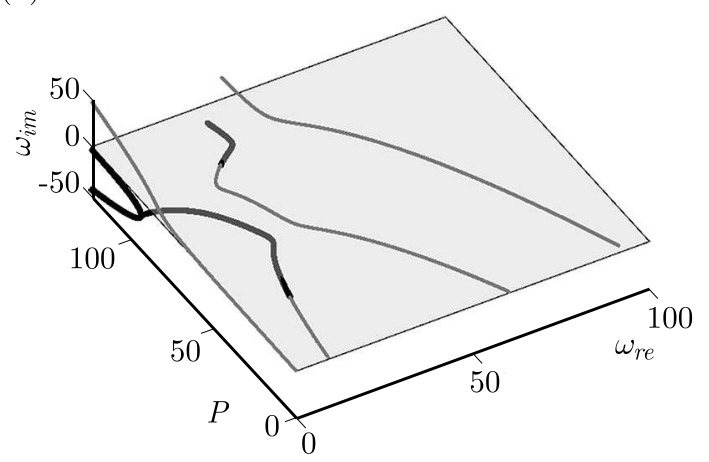

Fig. 5. Combined uniform and concentrated damping

It should be noticed that there is a change in the root curve of the imaginary part of the characteristic determinant on the left part of Fig. 5. On the right part, the complex eigenfrequencies are shown, both real and imaginary parts, for the same parameters of the damping. It is evident that the two arcs of the first branch, the growing and falling one, previously laying in the same plane, do not even touch. The growing part drops below the zero-plane of the imaginary part, $\omega_{i m}=\Im(\omega)=0$, before the maximum is reached. In fact, the maximum of that path does not exist - it appears just in the projection to the real part. Summing up, we can state that the approach using complex eigenvalue problems gives a deeper understanding of the dynamics of the considered construction, while the determinant-based solution gives quicker evaluations of the critical loads, e.g. during optimization.

\subsection{Hinged columns}

Reshaping the width of a column, using many segments or smooth variation, may be undesirable for several reasons. Nota bene, also the applicability of the Bernoulli-Euler theory is limited in the case of jumps in the column diameter. Instead of reducing the diameter, a reduction of the bending stiffness may be obtained as well by connecting two segments by a flexible joint, cf. for example (Bogacz et al., 2008). Using its position and compliance as design parameters, it can be attempted to increase critical loads.

In Fig. 6, the root curves for a column composed of two segments, the lower part of length $0.9 l$, the upper of length $0.1 l$, is shown. Both segments have identical and uniform mass density and stiffness. They are connected by a hinge with a bending stiffness of 1.0 on the left part of Fig. 6 . A $20 \%$ increase in the compliance causes considerable changes in the resonance curves, as is shown on the right part. The solution is very sensitive to small changes of the position of the hinge and the stiffness. The obtained increase in $P_{c r}$ of around $20 \%$ may be lost if, e.g. due to fatigue, the optimal parameters are not kept with sufficient accuracy. On the right part of Fig. 6, the improvement on $P_{c r}$ is already reduced by $20 \%$, and it quickly drops below the original level of $P_{c r}=20.05$, if the stiffness of the hinge decreases further.

Thus, hinges should be studied in connection with lateral supports, as discussed in the previous Subsection. Of particular interest are intelligent supports that are neither elastic nor 
(a)

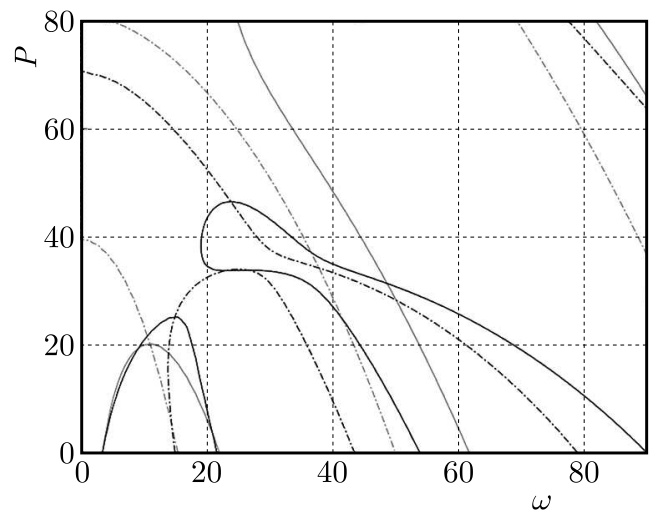

(b)

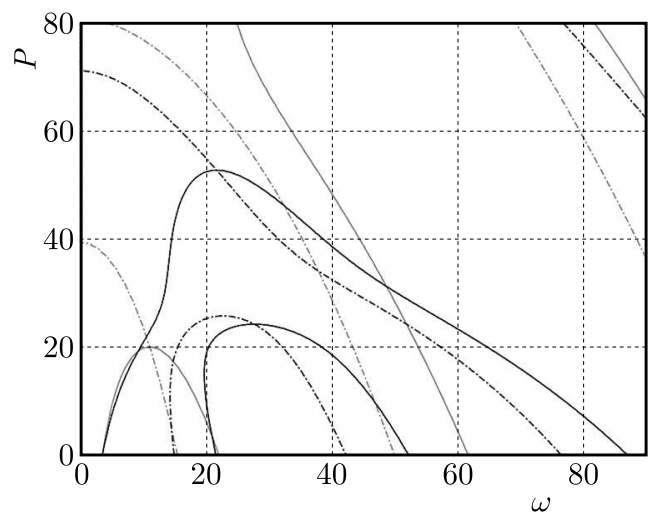

Fig. 6. Characteristics of a hinged column

viscous, but controlled on the basis of observed displacements and speeds. For a detailed analysis, we refer to (Bogacz et al., 2008), in the case of control see also (Preumont and Seto, 2008; Przybyłowicz, 2008).

\section{Conclusions}

Considerations on the stability of constructions should take into account dynamical effects. Static analysis alone can be very misleading. Secondly, one should be aware that loads may vary if a body gets out of its position of rest; such changes may have a stabilizing or destabilizing effect. Once the limits of stability are calculated, usually efforts will be made to improve the structure. However, effects of changes of the structure or the loading may be very surprising. For instance, weakening by removal of the material and decreasing the bending stiffness may increase the critical load, since it also reduces the inertial forces. Indeed, there may be unexpected crosseffects. If applied in the right region of the body, material may be saved and/or higher critical loads may be achieved. Similarly, adding damping may increase or decrease the critical force, depending on the geometrical details. If combined with mechatronic control, even a hinge may have a positive influence on the stability limit for the compressive load.

Finally, it should be warned that whatever optimized structures are designed and implemented, they should be robust against disturbed parameters and conditions. Such disturbances may be small changes of mass density (e.g. by dirt, corrosion), of stiffness (e.g. by ageing, fatigue, heat) or the loading (e.g. by wind, earthquakes) or a breakdown of the control system (e.g. power outing, shortcut, wear). In a recent paper, the robustness with respect to limited geometrical accuracy was discussed. From the analysis there, it follows that the limit of stability drops by $50 \%$ due to the change from the true theoretically optimal shape to a realistic one that can really be built. Anyway, given the theoretical potential to lift the critical load by a factor of seven, eight or more, there remains quite a good possibility to improve a construction at a low cost and in a save way.

\section{References}

1. BECK M., 1952, Die Knicklast des einseitig eingespannten tangential gedrehten Stabes, ZAMP Zeitschrift für angewandte Mathematik und Physik, 3, 3, 225-228

2. Bogacz R., CzyczuŁa W., Konowrocki R., 2014, Influence of sleepers shape and configuration on track-train dynamics, Shock and Vibration, art. ID 393867, 1-7

3. Bogacz R., Frischmuth K., 2012, On some new aspects of contact dynamics with application in railway engineering, Journal of Theoretical and Applied Mechanics, 60, 1, 119-129 
4. Bogacz R., Frischmuth K., Lisowski K., 2008, Interface conditions and loss of stability for stepped columns, Applied Mechanics and Materials, 9, 41-50

5. Bogacz R., Irretier H., Mahrenholtz O., 1980, Optimal design of structures subjected to follower forces, Ingenieur-Archiv, 49, 63-71

6. Bogacz R., Janiszewski R., 1985, Analysis and synthesis of columns, compressed by follower forces, from the stability point of view (in Russian), Advances in Mechanics, 8, 3, 3-52

7. Claudon J.L., 1975, Characteristic curves and optimum design of two structures subjected to circulatory loads, Journal de Mécanique, 14, 3, 531-543

8. Euler L., 1778, Determinatio onerum, quae columnae gestare valent, Acta Academiae Scientiarum Petropolitanae, 1, 121-145

9. Euler L., 1778, Examen insignis puradoxi in theoria columnarum occurentis, Acta Academiae Scientiarum Petropolitanae, 1, 146-162

10. Feldt W.T., Nemat-Nasser S., Prasad S.N., Herrmann G., 1969, Instability of a mechanical system induced by an impinging fluid jet, Journal of Applied Mechanics, 693-701

11. Frischmuth K., Kosinski W., Lekszycki L., 1993, Free vibrations of finite memory material beams, International Journal of Engineering Science, 31, 3, 385-395

12. Hanaoka M., Washizu K., 1980, Optimum design of Beck's column, Computers and Structures, 11, 6, 473-480

13. ImieŁowski S., Mahrenholtz O., 1997, Optimization and sensitivity of stepped columns under circulatory load, Applied Mathematics and Computer Science, 7, 1, 155-170

14. Kaliski S., Solarz L., 1962, Aeroelastic vibration and stability of a deformable rotating rocket in a linearized flow, Proceedings of Vibration Problems, 3, 57-68

15. Katsikadelis J.T., Tsiatas C.G., 2007, Nonlinear dynamic stability of damped Becks-column with variable cross-section, International Journal of Nonlinear Mechanics, 42, 1, 164-171

16. KERR A.D., 1988, Stability of a water tower, Ingenieur-Archiv, 58, 428-436

17. Kosiński W., Frischmuth K., 2001, Thermomechanical coupled waves in a nonlinear medium, Wave Motion, 34, 2, 131-141

18. Preumont A., Seto K., 2008, Active Control of Structures, Wiley

19. PrzybyŁowicz P.M., 2008, Aeroflutter suppression in an FGM panel with active fibers, Mechanics and Mechanical Engineering, 12, 4, 375-387

20. Ringertz U. T., 1994, On the design of Beck's column, Structural Opimization, 8, 120-124

21. Tada Y., Matsurnoto R., Oku A., 1989, Shape determination of nonconservative structural systems, Proceedings of 1st International Conference of Computer Aided Optimum Design of Structures: Recent Advances, Springer, Berlin

22. Tada Y., Seguchi Y., Kema K., 1985, Shape determination of nonconservative structural systems by the inverse variational principle, Memoirs of the Faculty of Engineering, Kobe University, 32, 45-61

23. Timoshenko S., 1921, On the correction for shear of the differential equation for transverse vibrations of prismatic bars, Philosophical Magazine, 6, 41, 744-746

24. Tomski L., Przybylski J., GoŁębiowska-Rozanow M., Szmidla J., 1996, Vibration and stability of an elastic column subject to a generalized load, Archive of Applied Mechanics, 67, $105-116$ 